Cuadernos de Relaciones Laborales

ISSN: $1131-8635$

http://dx.doi.org/10.5209/CRLA.59555

\title{
La representación sindical en España: cobertura y límites*
}

\author{
Pere Jódar ${ }^{1}$; Ramon Alós ${ }^{2}$; Pere Beneyto ${ }^{3}$; Sergi Vidal ${ }^{4}$
}

Recibido: 17 de octubre de 2016 / Aceptado: 01 de diciembre de 2016

Resumen. El objetivo de este artículo es realizar una aproximación al sistema de representación unitaria en la empresa en España a partir de una explotación estadística de los datos de las elecciones sindicales. Se trata de conocer aspectos como el alcance de la representación unitaria, la participación de los asalariados y la audiencia electoral de los sindicatos, con el objetivo de delimitar su representatividad y legitimidad. Nuestro trabajo se centra en el análisis de los tres últimos períodos electorales (2003-2007, 2007-2012 y 2012-2015), con objeto de evaluar el impacto del ciclo económico sobre la representación de los asalariados.

Palabras clave: sindicalismo; elecciones sindicales; representación unitaria en la empresa

\section{[en] Trade union representation in Spain: coverage and limits}

\begin{abstract}
The objective of this article is to make an approach to the unitary representation system in the company in Spain, from a statistical exploitation of union elections data. The analysis aims to discover aspects such as the scope of the unitary representation, participation of employees and unions electoral audience, in order to define their representativeness and legitimacy. Our work focuses on the analysis of the last three election periods (2003-2007, 2007-2012 and 2012-2015), in order to assess the impact of the economic cycle on the representation of workers.
\end{abstract}

Key words: unionism; union elections; workers' representation

Sumario. 1. Introducción. 2. Metodología. 3. Resultados. 3.1. Las empresas y la representación sindical en el centro de trabajo. 3.2. Participación de los trabajadores. 4. La presencia de diferentes opciones sindicales. 5. Discusión y conclusiones. 6. Bibliografía.

Cómo citar: Jódar, P.; Alós, R.; Beneyto, P.; Vidal, S. (2018) "La representación sindical en España: cobertura y límites". Cuadernos de Relaciones Laborales, 36(1), 15-34.

Este trabajo forma parte de un estudio más amplio para la Fundación $1^{\circ}$ de Mayo, de CCOO, en el que además de los firmantes ha participado Oscar Molina.

1 Pere Jódar, profesor de la Universidad Pompeu Fabra, Departamento de Ciencias Políticas y Sociales (pere. jodar@upf.edu)

2 Ramon Alós, investigador del Centro de Estudios Sociológicos sobre la Vida Cotidiana y el Trabajo (QUIT) e Instituto de Estudios del Trabajo (IET) (ramon.dealos@uab.cat)

3 Pere Beneyto, profesor de la Universidad de Valencia, Departamento de Sociología (pere.j.beneyto@uv.es)

4 Sergi Vidal, Centre d’Estudis Demogràifics. Universitat Autonòma de Barcelona (svidal@ced.uab.es) 


\section{Introducción}

La representación democrática de los trabajadores en España es relativamente reciente; y no sólo tiene que ver con las iniciativas de los asalariados o de sus organizaciones, ni tampoco con la interacción de éstas con el resto de agentes de las relaciones laborales (patronal y gobiernos), dado que en gran medida se ha consolidado fruto de decisiones legislativas que condicionan el conjunto de su marco institucional. Dichas iniciativas legales comenzaron en plena transición democrática, en primer lugar, mediante una regulación provisional (Ley 19/1977, de 1 de abril, de Asociación Sindical; Real Decreto 3149/1977, de 6 de diciembre, sobre elección de delegados y comités de empresa) y, de forma definitiva, a partir de la Constitución (arts. 7 y 28.1), el Estatuto de los Trabajadores (ET, 1980) y la Ley Orgánica de Libertad Sindical (LOLS, 1985). Sin olvidar que en las decisiones legislativas pesaron no sólo las necesidades derivadas del cambio de régimen, sino también las presiones de la crisis económica y las decisiones del bloque hegemónico. El resultado de este proceso fue un sistema de doble canal, asociativo (afiliación) y electivo (representación delegada), cuya articulación está regulada legal y sindicalmente, a tres niveles:

Empresa: la interlocución y negociación con la dirección de la misma corresponde a la representación unitaria de los trabajadores; aunque para las grandes compañías la LOLS regula los derechos correspondientes a las secciones sindicales o sindicato en la empresa (canal asociativo). La coexistencia de ambos canales no siempre es fácil y genera problemas y debates de interés (free rider, etc.).

Sectorial: están legitimados para la negociación colectiva los sindicatos en función de su representatividad electoral; de manera que ésta se define a partir de un criterio general, aplicable a cualquier ámbito local y sectorial, en función del número de delegados que en representación de los trabajadores obtiene cada sindicato en las empresas del ámbito (sector, territorio) al que se hace referencia.

Institucional: los sindicatos que acrediten electoralmente su representación mayoritaria (un mínimo del 10\% de la representación en el ámbito de referencia o un $15 \%$ si se trata de organizaciones autonómicas) tienen reconocida su participación en instancias públicas de consulta (Consejo Económico y Social, Seguridad Social, Fundación Tripartita para la formación, etc.) y en los procesos de diálogo social.

Se trata de un sistema con voluntad inclusiva. Se implantó en su momento ante el vacío que podía comportar la transición de la dictadura a la nueva democracia (Baylos, 1991). Este sistema, por una parte, parece desincentivar la afiliación al universalizar la cobertura de la intervención sindical; pero, por otra, contribuye a ampliar su área de influencia por la vía de la representación electoral, al tiempo que le aporta legitimidad democrática, mediante el proceso electoral y generaliza las garantías fruto de la negociación colectiva. De ahí que la actual ofensiva neoliberal ponga tanto empeño en desregular y descentralizar la negociación colectiva y deslegitimar a los sindicatos. Se trata, asimismo, de un sistema con rasgos neocorporatistas dado que la representatividad obtenida mediante elecciones potencia la legitimación de los sindicatos más votados, pero también su presencia en la negociación colectiva, 
con la pretensión de favorecer acuerdos y pactos sociales. Éstos se han celebrado en numerosas ocasiones, pero han tenido un impacto relativo en las relaciones laborales españolas. En primer lugar, porque los sucesivos gobiernos han preferido en muchas ocasiones la vía legislativa; y en segundo lugar porque en España se ha mantenido un sistema de relaciones laborales de oposición entre los agentes, manteniendo el Estado una mediación con preferencia por las demandas empresariales, lo que ha dado lugar a un sistema de conflictividad laboral elevada respecto de Europa y con convocatorias sucesivas de huelga general. Dicha movilización tuvo éxito en el período comprendido entre 1988 y 2002, pero como en el resto de Europa, los gobiernos tras 2008 han contemplado con indiferencia su celebración, mientras han ampliado la vía del decreto ley, intentando dar un golpe de mano a la acción autónoma de las partes en el campo de las relaciones laborales. Sintomático de todo ello es el hecho de que a pesar de que la legislación marca claramente que la representatividad sindical en España se basa en elecciones, la patronal, el gobierno y los think thanks de la derecha reclaman la representación afiliativa.

En todo caso el marco legal estableció que el reconocimiento del derecho a la representación unitaria de los trabajadores en todas las empresas o centros de trabajo, comenzaba en el límite inferior de 6 trabajadores (art. 62.1, ET), siempre que un tercio de los mismos o que un sindicato representativo solicite su convocatoria, o bien se decide por mayoría cuando cuentan entre 6 y 10 trabajadores. En estas elecciones se presentan las candidaturas formadas por trabajadores de la misma empresa, en listas habitualmente sindicales, siendo electores todos los trabajadores en plantilla que lleven al menos un mes en la empresa. El número de representantes a elegir depende del tamaño de la plantilla (tabla 1) y, como veremos, la decisión tomada por el legislador en su momento tendrá una fuerte influencia en el conjunto.

Tabla 1. Número de representantes a elegir según dimensión de la empresa o centro de trabajo, en España

\begin{tabular}{|ll|}
\hline & Representantes \\
\hline 6 a 30 trabajadores & 1 \\
\hline 31 a 49 trabajadores & 3 \\
\hline 50 a 100 trabajadores & 5 \\
\hline 101 a 250 trabajadores & 9 \\
\hline 251 a 500 trabajadores & 13 \\
\hline 501 a 750 trabajadores & 17 \\
\hline 751 a 1000 trabajadores & 21 \\
\hline Más de 1000 trabajadores & 2 por fracción de mil, con un máximo de 75 \\
\hline
\end{tabular}

Desde la primera convocatoria de elecciones sindicales a principios de 1978, anterior incluso a la regulación normativa por el ET, la evolución de su cobertura y resultados, refleja tanto las oscilaciones del cambio económico y ocupacional, como la recomposición interna del movimiento sindical, en períodos que siguen al inicio los avatares políticos y, después, los ciclos económicos. La tabla 2 nos permite contemplar dichas incidencias en la evolución del número de delegados, así como una aproximación de la competencia electoral entre las diferentes opciones sindicales. Primero, y en general, CCOO y UGT atraviesan por sucesivas fases de "sorpasso" 
mutuo, hasta que a finales de los 90, aunque con ligera ventaja de CCOO, siguen líneas bastante paralelas. En segundo lugar, se confirma la hegemonía del tándem UGT-CCOO respecto del resto de opciones; su punto máximo en términos de representatividad se alcanza en 1990, consolidándose posteriormente en torno al 70-75\%, con una ligera tendencia a la baja en los últimos años.

Tabla 2. Elecciones sindicales en España (1978-2015)

\begin{tabular}{|c|c|c|c|c|c|c|c|c|c|c|}
\hline \multirow[t]{2}{*}{ AÑO } & \multirow[t]{2}{*}{ Asalariados* } & $\begin{array}{c}\text { TOTAL } \\
\text { DELEGAS- } \\
\text { DOS }\end{array}$ & \multicolumn{2}{|c|}{$\mathrm{CCOO}$} & \multicolumn{2}{|c|}{ UGT } & \multicolumn{2}{|c|}{ Otros } & \multicolumn{2}{|c|}{ No sindicales } \\
\hline & & & $\mathrm{N}^{\circ}$ & $\%$ & $\mathrm{~N}^{\circ}$ & $\%$ & $\mathrm{~N}^{\circ}$ & $\%$ & $\mathrm{~N}^{\mathrm{o}}$ & $\%$ \\
\hline 1978 & $8.479,2$ & 193.112 & 66.540 & 34,5 & 41.897 & 21,7 & 25.953 & 13,4 & 58.725 & 30,4 \\
\hline 1980 & $8.028,8$ & 164.617 & 50.817 & 30,8 & 48.194 & 29,3 & 22.053 & 13,4 & 43.553 & 26,5 \\
\hline 1982 & $7.681,7$ & 140.770 & 47.016 & 33,4 & 51.672 & 36,7 & 25.058 & 17,8 & 17.024 & 12,1 \\
\hline 1986 & $7.675,3$ & 175.363 & 59.230 & 33,8 & 69.427 & 39,6 & 33.998 & 19,4 & 12.708 & 7,2 \\
\hline 1990 & $9.273,4$ & 237.261 & 87.730 & 36,9 & 99.737 & 42,0 & 41.387 & 17,4 & 8.407 & 3,5 \\
\hline 1995 & $8.942,7$ & 204.586 & 77.348 & 37,8 & 71.112 & 34,7 & 49.495 & 24,2 & 6.631 & 3,2 \\
\hline 1999 & $10.668,6$ & 260.285 & 98.440 & 37,8 & 96.770 & 37,2 & 57.006 & 21,9 & 8.969 & 3,1 \\
\hline 2003 & $12.433,6$ & 283.075 & 110.208 & 38,9 & 103.805 & 36,7 & 69.062 & 24,4 & -- & -- \\
\hline 2007 & $16.968,1$ & 312.017 & 122.079 & 39,1 & 114.973 & 36,8 & 74.965 & 24,0 & -- & -- \\
\hline 2012 & $14.573,4$ & 303.622 & 113.721 & 37,5 & 107.459 & 35,4 & 75.788 & 24,9 & 6.654 & 2,2 \\
\hline 2015 & $14.773,5$ & 259.282 & 93.662 & 36,1 & 85.254 & 32,9 & 73.956 & 28,5 & 6.410 & 2,5 \\
\hline
\end{tabular}

Fuente: con datos del Ministerios de Trabajo y AAPP para los datos de 1978 a 1990, SIGIS-CCOO, para las convocatorias de 1995 a 2007 y Ministerio de Empleo para 2012 y 2015.

(*) INE: medias anuales (en miles).

Las cuatro primeras convocatorias de elecciones sindicales se celebraron sólo en empresas del sector privado, mientras que a partir de 1987 el sistema se amplió, con algunas peculiaridades formales (distinta definición de las unidades electorales y distribución de las Juntas de Personal), a los funcionarios de la Administración Pública, Sanidad, Enseñanza, Justicia, etc., tras la aprobación de la LOR ${ }^{5}$, lo que aumentó notablemente su cobertura y representatividad. Aunque el hecho de que una parte sustancial de los asalariados españoles (función pública) no adquiriera una relativa normalidad representativa hasta diez años después del fin de la dictadura muestra parte de los condicionamientos que han pesado sobre el sistema de relaciones laborales español.

Otra modificación importante en la regulación formal e implementación práctica del sistema de representación fue la derivada del Acuerdo Interconfederal entre CCOO y UGT, de septiembre de 1992, por el que se suprimió el período cerrado

Ley 9/1987, de 12 de junio, de Órganos de Representación, Determinación de las condiciones de trabajo y Participación del personal al servicio de las Administraciones Públicas. 
de cómputo de tres meses cada cuatro años para la realización de las elecciones sindicales y se fijaba un sistema de cómputo dinámico de actualización continua de resultados electorales. Esto contribuyó a rebajar considerablemente la concurrencia cuasi-política de los sindicatos participantes. A partir de dicha reforma legal se eliminó, además, la proclamación global de resultados, fijándose un sistema de acreditación en función del ámbito (convenio, sector, territorio, etc.) y del momento en que se ejerce la representatividad derivada de los mismos.

En cuanto a los resultados globales y por candidaturas (tablas 2 y 3), si bien la información oficial disponible no ofrece un alto grado de desagregación sí aporta datos globales relevantes que permiten identificar algunas tendencias, entre las que destacan:

Incremento de la representación: tras cada fase de ciclo económico recesivo, ha experimentado un auge considerable. Véase la diferencia entre el número de delegados entre 1982 y 1986, y entre 1995 y 1999. En la actualidad la fase de declive económico y ocupacional iniciada en 2007 repercute en negativo, pero habrá que estar atentos a lo que suceda al final del ciclo.

Mayoría de CCOO: con el 36,1\% del total de los delegados electos en 2015, CCOO se confirma como primera fuerza sindical, con 3,2 puntos porcentuales y 8.408 delegados electos por encima de UGT, consolidando una tendencia iniciada en 1995.

Sindicatos más representativos: conforme a la normativa vigente tan sólo CCOO y UGT alcanzan la condición de organizaciones "más representativas" a nivel general, por superar el 10 por cien de los delegados electos, acumulando entre ambas el 69\% de la representatividad en 2015, tras incrementarse notablemente desde 1978 (56,2\% de la representación) hasta 2007; aunque tras esa fecha se modera. También ELA en el País Vasco $(40,1 \%)$ y Navarra (21,9\%), LAB (18,9\%) en el País Vasco y CIGA (27,7\%) en Galicia obtienen por sus resultados autonómicos el reconocimiento de sindicatos "más representativos".

Fragmentación de los sindicatos minoritarios: además de los sindicatos reconocidos como más representativos a nivel estatal y en las CCAA del País Vasco, Navarra y Galicia, se registra un grupo heterogéneo de sindicatos minoritarios (hasta un total de 514 en el último proceso electoral) que, en su conjunto, acumulan un total de 57.499 delegados electos, equivalente al 22,1\% del total. Con la excepción de USO (que ocupa el tercer lugar en el conjunto, aunque representa el 3,9\%) y de CGT (con el 1,9\%), se trata en general de sindicatos corporativos o de empresa, implantados en algunos sectores y/o niveles de la función pública y otros servicios como grandes almacenes (FETICO, FASGA), enseñanza (FSIE, STES, ANPE), empresas públicas privatizadas, etc.

Sindicalización de la representación: sólo 6.410 delegados $(2,5 \%$ del total en 2015, frente a un 30,4\% en 1978) han sido elegidos en candidaturas no vinculadas a ningún sindicato. Esto supone que se ha completado prácticamente el ciclo de sindicalización iniciado en la segunda mitad de los años ochenta, tras una primera fase en la que los no adscritos suponían más de una cuarta parte de los electos. Esta evolución contrasta con la de países como Estados Unidos o Reino Unido, que muestran una tendencia contraria. 
Tabla 3.- Representación sindical de asalariados y funcionarios, 2015

\begin{tabular}{|c|c|c|c|c|c|c|}
\hline & \multicolumn{2}{|c|}{ Asalariados* } & \multicolumn{2}{c|}{ Funcionarios } & \multicolumn{2}{c|}{ Total } \\
\hline & Núm. & $\%$ & Núm. & $\%$ & Núm. & $\%$ \\
\hline CCOO & 89.108 & 37,4 & 4.554 & 21,4 & 93.662 & 36,1 \\
\hline UGT & 81.402 & 34,2 & 3.852 & 18,1 & 85.254 & 32,8 \\
\hline USO & 9.774 & 4,1 & 491 & 2,3 & 10.265 & 3,9 \\
\hline CSIF & 6.171 & 2,6 & 3.700 & 17,4 & 9.871 & 3,8 \\
\hline ELA-STV & 7.555 & 3,2 & 554 & 2,6 & 8.109 & 3,1 \\
\hline CGT & 4.439 & 1,9 & 501 & 2,4 & 4.940 & 1,9 \\
\hline FETICO & 4.304 & 1,8 & -- & -- & 4.304 & 1,7 \\
\hline CIG & 3.879 & 1,6 & 367 & 1,7 & 4.246 & 1,6 \\
\hline LAB & 3.693 & 1,6 & 409 & 1,9 & 4.102 & 1,6 \\
\hline Otros & 21.510 & 9,0 & 6.599 & 31,0 & 28.119 & 10,8 \\
\hline No afiliados & 6.169 & 2,6 & 241 & 1,1 & 6.410 & 2,5 \\
\hline TOTAL & 238.014 & 100 & 21.268 & 100 & 259.282 & 100 \\
\hline
\end{tabular}

Fuente: Ministerio de Empleo

(*) Sector privado y personal laboral de las Administraciones Públicas

Distribución sectorial: aun registrándose algunas pautas diferenciadas entre asalariados y funcionarios (mayor fragmentación y presencia de las opciones corporativas, en el segundo caso), la titularidad (pública o privada) de los centros de trabajo no ha generado en nuestro país una fractura profunda en la representación sindical (vid. Tabla 3). CCOO y UGT mantienen su mayoría relativa entre los funcionarios, aunque en proporción sensiblemente inferior a la del sector privado (39,5 y 71,6\%, respectivamente), pese a las pretensiones hegemonistas impulsadas inicialmente por CSIF (2,6\% sobre el total de delegados electos y $17,4 \%$ entre los funcionarios) para el conjunto de la Administración Pública y por otras organizaciones corporativas (STEs, ANPE, CEMSATSE, etc.) en subsectores como la enseñanza y la sanidad.

Se trata, pues, de un sistema de representación caracterizado por un importante grado de cobertura y de homogeneidad. Sin embargo, mantiene puntos débiles. La representación mayoritaria se concentra en los dos grandes sindicatos de clase (CCOO y UGT), que mantienen, además, desde hace dos décadas una estrategia de unidad de acción consolidada que se proyecta en la negociación colectiva y en la representación institucional; pero existe una pluralidad de sindicatos que pueden condicionar la representación y la negociación en grandes empresas y sectores determinados. Otra debilidad es la representación de los segmentos precarios, sobre todo de la pequeña empresa y de los sectores de actividad más intensivos en mano de obra. 


\section{Metodología}

Nuestra aproximación a las elecciones sindicales en España tiene en cuenta diferentes variables del contexto: dimensión y dinámica de la empresa, sectores económicos, centros de trabajo; además, nuestra perspectiva se orienta por los resultados de los procesos electorales estudiados en diferentes países por, entre otros, Farber (2001) y Charlwood y Forth (2011).

Naturalmente analizamos la representación de los asalariados españoles por medio de las opciones sindicales presentes (o en competición), así como en relación a la afiliación. En todo caso, las oportunidades ofrecidas por los sindicatos introducen variaciones a la influencia que ejercen los factores estructurales; aunque es evidente que existe una interacción entre éstos con las organizaciones de los trabajadores, y no sólo un estímulo de sentido único. Aquí son clave las sugerencias de Taras y Kaufman (2006), Charlwood y Forth (2011) o Artus (2013).

Asimismo, tenemos en cuenta la existencia de factores transversales, en los que la estructura, la propensión individual y las oportunidades organizativas pueden o no coincidir, contribuyendo a la variación de los resultados; por ejemplo, el territorio (comunidad autónoma) y las variables demográficas (sexo, edad). Los resultados de Blaschke (2011), Le Capitaine et al. (2011) o Artus (2013), permiten contrastar nuestros hallazgos.

El hilo conductor de esta investigación ${ }^{6}$ tiene como hipótesis-guía la complementariedad de las formas de representación (sindical, unitaria, etc.), en un sistema de doble canal y con fuerte regulación legal e intervencionismo estatal -reformas laborales- (Gumbrell-McCormick y Hyman, 2006; Rigby et al., 2009; Freeman, 2007).

Los datos utilizados son los oficiales proporcionados por el Ministerio de Empleo (negociación colectiva) o de la Seguridad Social (trabajadores y empresas), así como los resultados de los procesos electorales facilitados por el propio Ministerio y por el Servicio de Información Confederal (SIC) de CCOO, coincidentes en términos generales, si bien con pequeñas diferencias en función de la fecha de registro. Esto nos ha permitido algunas desagregaciones que en el artículo solo podemos ofrecer sucintamente; así como comparar en ocasiones la representación electoral de los trabajadores con la afiliación sindical. También hemos utilizado los datos de la última Encuesta de Calidad de vida en el Trabajo, correspondiente a 2010, para realizar algunas puntualizaciones. La información recogida excluye a los funcionarios y los resultados de las elecciones efectuadas en su ámbito; ello es debido a que la unidad de análisis es la empresa y dicha información tiene origen en los ficheros de la Seguridad Social, que no contemplan el ámbito funcionarial. La base de datos es enorme ya que trabajamos con las informaciones referidas a cuatro años (finales de 2003, 2007, 2012 y 2015) y requirió un arduo trabajo de selección, contrastación, ordenación y clasificación. 


\subsection{Resultados}

Este apartado de resultados se inicia con una panorámica general, incluyendo algunas concreciones de la representación electoral de los trabajadores en España, basados en la ECVT. Ello nos permite puntualizar algunos aspectos cruciales de dicha representación que, a menudo, voces interesadas confunden; esto es la diferencia entre presencia (afiliación), audiencia (elección) e influencia (cobertura de la negociación colectiva y capacidad de movilización). A partir de ahí presentamos los resultados basados en la base de datos comentada, ordenándolos en tres subapartados: empresas y centros de trabajo; participación de los trabajadores; y, finalmente, representación de las diferentes opciones sindicales.

Es posible una aproximación a la cobertura de la representación sindical a través de la información aportada por la Encuesta de Calidad de Vida en el Trabajo (ECVT), realizada por el Ministerio de Trabajo desde 1999 e interrumpida en 2010. Se observa que dicha cobertura era del $47,4 \%$ sobre el total de la población asalariada, lo que equivale, aproximadamente, a 7.100.000 trabajadores, tras un crecimiento sostenido que en dicha década fue especialmente importante. Se trata de valores mínimos, pues en el $15,3 \%$ de los casos no consta una respuesta clara al respecto.

Tabla 4. Evolución de la afiliación y representación sindical en España (2000-2010)

\begin{tabular}{|l|l|l|l|}
\hline & 2000 & 2010 & Evolución 2000-10 \\
\hline $\begin{array}{l}\text { Afiliación } \\
\text { Total }\end{array}$ & $\begin{array}{l}2.093 .500 \\
16,6 \%\end{array}$ & $\begin{array}{l}2.894 .200 \\
18,9 \%\end{array}$ & $+800.700(+38,2 \%)$ \\
Tasa & & & \\
\hline $\begin{array}{l}\text { Cobertura de la representación sindical } \\
\text { Total }\end{array}$ & $\begin{array}{l}5.400 .000 \\
\text { Tasa }\end{array}$ & $\begin{array}{l}7.100 .000 \\
47,4 \%\end{array}$ & $+1.700 .000(+31,5 \%)$ \\
\hline
\end{tabular}

Fuente: Ministerio de Empleo, Encuesta de Calidad de Vida en el Trabajo

Los datos de la ECVT 2010 permiten desagregar la cobertura de la representación sindical, de manera que se observa su aumento con el tamaño de la empresa, o su distribución desigual según sectores de actividad, desde niveles inferiores a la media $(47,4 \%)$ en los más fragmentados, con empleo precario y golpeados por la crisis (agricultura, construcción y servicios personales) hasta cotas notablemente más altas en la industria $(57,1 \%)$, transportes y comunicaciones $(59,7 \%)$, servicios financieros $(64,3 \%)$ y servicios públicos $(61,5 \%)$.

Especialmente significativa resulta la relación entre la afiliación (presencia) y la representación sindical (audiencia), que por una parte eleva la tasa media de afiliación (del 18,9\% hasta el 30\% en 2010) entre los trabajadores que cuentan con representación elegida en sus empresas, y la rebaja hasta el 9,6\% entre quienes no disponen de la misma. Así como, correlativamente, la cobertura de la representación crece hasta el 75,4\% entre quienes declaran estar afiliados y desciende hasta el 40,9\% entre quienes no lo están. También aparece una correspondencia bastante precisa entre trabajadores que cuentan con representación sindical, la tasa 
de afiliación y el grado de información que tienen los asalariados sobre el convenio colectivo (fruto de dicha representación). Por ejemplo, todos los porcentajes asociados con estas variables aumentan con el incremento del tramo de plantilla de la empresa; asimismo, son más elevados en los sectores de servicios financieros, transportes y comunicaciones, industria o servicios públicos. El tipo de contrato también muestra la diferencia entre indefinidos y temporales en todos estos conceptos. Del mismo modo, los segmentos con mayor representación también tienen más afiliación (y viceversa): hombres, maduros, con estudios secundarios y universitarios, técnicos, del sector público. Sólo se aprecia variación en la ocupación: los trabajadores manuales cualificados y no cualificados, tienen más afiliación que los administrativos que, en cambio, cuentan con mayor representación sindical. En todo caso los segmentos de trabajadores más divididos, pequeña empresa, sectores fragmentados, precarios, obtienen porcentajes bajos tanto de representación, como de afiliación e información.

Cabe señalar que, junto a la afiliación directa y la representación electoral, otro indicador cuantificable de la intervención sindical en nuestro país es el de la cobertura de la negociación colectiva que, según la legislación vigente ejercen los sindicatos en función de la representatividad acreditada.

La negociación de los convenios colectivos corresponde a los representantes electos a nivel de empresa (delegados y Comités) cuando se trata de convenios de este ámbito (con datos provisionales para 2015: 4.206, para un total de 808.300 trabajadores) y a los sindicatos más representativos para los de ámbito superior (990 convenios y 8.273.300 trabajadores), lo que se traduce en una distribución de la representación concentrada mayoritariamente en torno a CCOO y UGT, que asumen la interlocución y defensa de la práctica totalidad de los trabajadores. Sin embargo, la aplicación de la reforma laboral (Ley 3/2012) puede suponer una caída en la cobertura del sistema, como exponen Baylos (2012 y Valdés Dal-Ré (2013), pero los datos de cobertura son durante largo tiempo provisionales y habrá que esperar para observar la tendencia real.

La participación sindical en la negociación colectiva mantiene con la afiliación y la representatividad relaciones complejas que oscilan entre la contradicción aparente y la complementariedad real, pues si bien es cierto que la eficacia universal de lo pactado en convenio colectivo por los sindicatos permite visualizar su capacidad de influencia, plantea también problemas al activar la lógica de delegación y desincentivar la participación directa. Es éste un tópico de la teoría de la acción colectiva (Olson, 1992), según la cual la decisión de afiliarse a un sindicato se explica en términos de maximización de la utilidad y de la relación coste/beneficios, lo que plantea la paradoja del "gorrón" (free rider). Según ésta, en un colectivo que comparte intereses siempre existe una fracción considerable de individuos para quienes el esfuerzo (coste) de la acción a realizar para proteger dichos intereses (la acción sindical, en este caso) es mayor que la expectativa de obtener resultados significativos de dicha acción (beneficios), puesto que éstos son públicos (lo reciben también quienes no se movilizan en defensa de los intereses comunes), mientras que los costes son individuales.

En el modelo español ello plantea paradojas ${ }^{7}$. Efectivamente, los beneficios de

La más importante es la que niega la propia base teórica de los presupuestos de Olson, dado que las organizaciones sindicales europeas no sólo son asociaciones profesionales (utilidad instrumental), sino que también cumplen una función social y democrática (valores solidarios); por otra parte, los trabajadores no sólo se afilian 
la concertación social, la negociación colectiva e, incluso, la intervención puntual de los representantes sindicales en la empresa o centro de trabajo, son de aplicación universal, mientras que los costes son asumidos en exclusiva por las organizaciones sindicales, sus afiliados y representantes; lo que al menos en una primera fase, y aún hoy en demasiados casos, opera como barrera de entrada a la afiliación, dada la beligerancia antisindical de muchas empresas y organizaciones, especialmente disuasoria para los status contractuales más frágiles y precarios. En cambio, el voto legitima al negociador y amplía su área de influencia sindical e, incluso, puede contribuir a la expansión afiliativa en determinadas empresas y sectores. Quizás por ello, desde los sindicatos, se plantea la necesidad de sindicalizar la representación y reforzar la presencia e intervención directa del sindicato en la empresa, asumiendo mayores niveles de visibilidad y de competencia y, por tanto, de implicación y participación de los trabajadores en las organizaciones sindicales de base.

Como ya se ha indicado anteriormente, según los datos de la ECVT-2010 existe una correlación positiva entre representación sindical y afiliación, de manera que la primera es la que tiene mayor influencia entre los determinantes de la afiliación (Köhler y Calleja, 2011). Aunque también, como hemos visto, la correlación opera en sentido contrario: a más afiliación, más representación. De este modo los datos de representatividad electoral y cobertura negociadora confirman suficientemente la legitimidad del sindicalismo español. En este sentido y para analizar la representación sindical distinguimos entre factores externos e internos. Los factores externos o de contexto (sector, empresa, ciclo económico) pueden influir en gran medida en la opción de los trabajadores; aunque pensamos que también actúan como variables institucionales debido a que la estructura legal de la representación de los trabajadores en España tiene su fundamento en la dimensión de las empresas. Asimismo, inciden factores internos, como la propensión de los asalariados hacia la participación, en este caso en forma de voto, así como las oportunidades que las organizaciones sindicales, con su acción colectiva, abren a dicha participación, especialmente mediante su presencia en la empresa o mediante adaptaciones organizativas que les acerque $o$ visibilice ante sus posibles electores.

\subsection{Las empresas y la representación sindical en el centro de trabajo}

El número total de empresas ${ }^{8}$ susceptibles de realizar elecciones aumentó en un 15,5\% en los años de expansión analizados (2003-2007) para reducirse en un 17,1\% con la crisis, siendo 287 mil a finales del año 2015. Sin embargo, las empresas que quedan excluidas del sistema electoral son una amplia mayoría (cerca de un millón en 2015), aunque en su mayor parte son unidades de uno o dos trabajadores.

por motivos instrumentales, sino también por defensa y protección, por valores o sociabilidad. Pero, en este artículo no corresponde profundizar en estos aspectos.

8 En realidad se trata de unidades de cotización a la Seguridad Social, esto es, empresas que desarrollan su actividad laboral en una misma provincia y que tienen características homogéneas frente a la cotización, que a efectos de simplificación denominamos como empresas. 
Gráfico 1. Proporción de empresas y de trabajadores en unidades de 6 o más trabajadores, España, diciembre de cada año

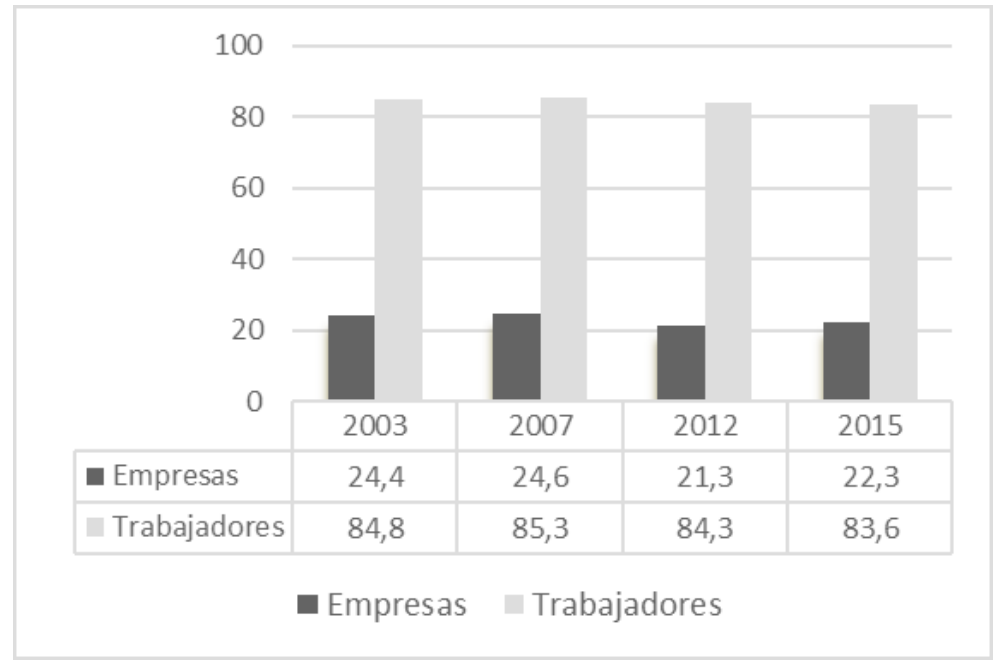

Fuente: Ministerio de Empleo y SS, Anuario de Estadísticas Laborales (varios años).

Por su parte, el número de trabajadores del sector privado (adscritos al régimen general y del carbón de la Seguridad Social) aumentó en un 18,4\% entre 2003 y 2007, descendiendo desde entonces en un 16,4\% hasta situarse a finales de 2015 en 12.305.873, de los que más de dos millones quedan excluidos del sistema de representación por trabajar en empresas de 1 a 5 asalariados. La dimensión de empresa, o centro de trabajo, junto con el sector, son variables fundamentales para comprender la dinámica de las elecciones sindicales y, por tanto, de la representación de los trabajadores en España.

La dispersión y fragmentación del sistema empresarial español es patente. Y ello supone dificultades y retos en términos de elecciones sindicales y, por tanto, de representación y más tarde de eficacia negociadora; pero también en términos de legitimación y consolidación del sistema sindical, en su función de contrapoder y mecanismo de control básico del sistema de relaciones laborales. No olvidemos que las relaciones laborales se asocian en términos económicos a la productividad y la competitividad, en términos laborales a la ocupación y su calidad y, en términos sociales, a la integración de los asalariados y la paz social. Posiblemente dicha fragmentación del sistema empresarial español, en la que hay una fuerte presencia de centros de trabajo de muy pequeñas dimensiones, explique mucho más los problemas de nuestro mercado de trabajo (segmentación, dualidad, precariedad, desempleo, ...), que no la continua reforma de la legislación laboral, con la ruptura de las reglas del juego de las relaciones entre los agentes y la precarización y empobrecimiento de las condiciones de trabajo de los asalariados. Por otra parte, un mayor tamaño del centro de trabajo supone más probabilidad de presencia sindical, de representación unitaria, de negociación sindical y de presencia de sindicatos no mayoritarios, también de capacidad de representación corporativa. Viceversa para el caso de las empresas de menor tamaño.

En términos de demografía empresarial, en el año 2003, el punto de partida eran 299.116 empresas de 6 o más trabajadores, que tras un proceso de intensa destruc- 
ción en los años posteriores a 2007 se convierten en 286.814 a finales de 2015. Los datos del SIC de CCOO permiten constatar informaciones de interés sobre la dinámica empresarial, aunque sólo hasta el año 2012. Así, tomando como referencia el año 2003, en 2012 se mantiene el 54,6\% de las empresas y, en el ínterin, se destruye el $45,4 \%$ de las mismas, creándose en el tiempo transcurrido 177.946 empresas, que equivalen al 60,9\% de las que había en el inicio. De las 177.946 empresas de nueva creación, en la etapa considerada, el 90,6\% son empresas sin representación sindical. Algunos autores advierten (Taras y Kaufman, 2006; Charlwood y Forth, 2011; Lévesque y Murray, 2010) que los cambios institucionales impulsados por gobiernos de tendencia neoliberal, junto a la actitud poco favorable de las empresas hacia los sindicatos, aumentan la probabilidad de acrecentar el fenómeno de la no representación o de la representación no sindical y, por tanto, de la desprotección de los trabajadores. Ahora bien, entre las empresas de reciente creación es más habitual que no se disponga de representación sindical, lo que revela una dinámica a tener en cuenta.

Estos cambios contextuales inciden en la representación de los trabajadores en las empresas de manera que la mayoría de ellas (de 6 o más trabajadores) no tienen representación; $75,0 \%$ en 2003 y $78,8 \%$ en 2015 . No obstante, las empresas sin representación sindical se concentran en los tramos de 6 a 30 trabajadores, que son el $83,4 \%$ de las empresas -excluyendo a las que tienen menos de 6 , que no realizan elecciones-.

Gráfico 2. Proporción de empresas y de trabajadores con representación sindical, en España (en \% sobre el total de unidades de 6 o más trabajadores)

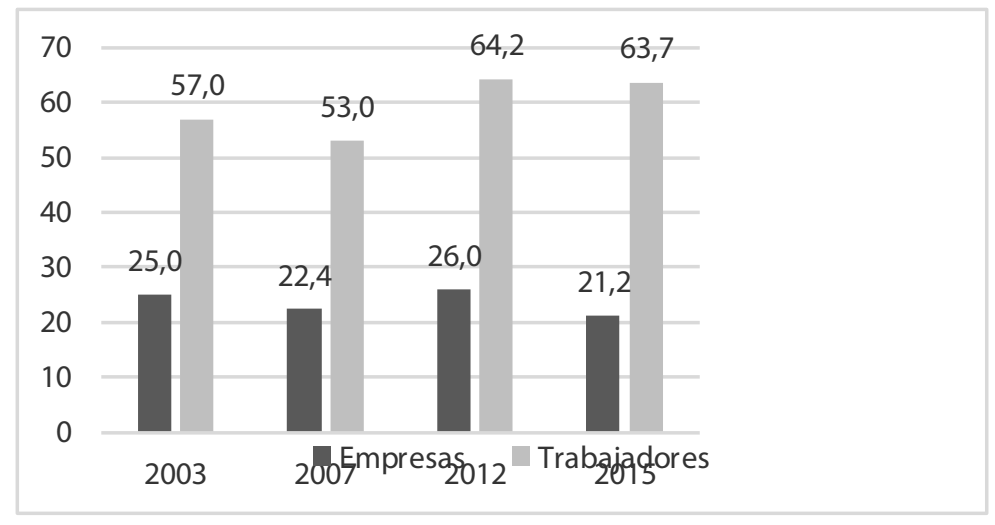

Fuente: con datos SIC-CCOO y de la Seguridad Social

No obstante, la ubicación de los asalariados en tanto que sujetos a representar presenta una extraordinaria diferencia con respecto a los valores anteriores; así, en 2015, poco más de 10 millones de trabajadores (el 83,6\%) de un total de algo más de 12 millones (referido al sector privado o laborales de las administraciones públicas), desempeñan su ocupación en empresas de 6 o más trabajadores y, por tanto, susceptibles de celebrar elecciones sindicales; casi el 65\% en 2015 trabajan en unidades de 50 o más trabajadores y cerca del 38\% en las 1.857 empresas de más de 500 asalariados. Y ya se ha visto que unos 2 millones de trabajadores $(15,5 \%$ 
del total) no tienen ningún derecho a representación. Mientras entre aquellos que sí podrían tener representación, el 37,3\% no cuenta con delegado o miembro de comité de empresa.

Una vez conocida la base de representación que establece que las elecciones sindicales se pueden celebrar en el $22,3 \%$ de las empresas (referido a 2015), para representar al 83,6\% de los asalariados, se observa que el 43,4\% de dichas empresas tienen de 6 a 9 trabajadores y hasta un 90,3\% menos de 50 asalariados. Por tanto, la dispersión y la enorme cantidad de pequeña y microempresa dificultan la institucionalización de las relaciones laborales en España.

Del mismo modo, la probabilidad de que las empresas no tengan representación sindical está asociada con la dimensión de sus plantillas. Si en las empresas de más de 250 trabajadores poco más del 10\% no cuenta con representación sindical, en las más pequeñas, de 6 a 9 trabajadores apenas un $7 \%$ cuenta con delegado sindical. En general, la probabilidad de que una empresa no tenga representación sindical es mayor cuando es de nueva creación, con muy poca plantilla, de actividades de servicios privados y sin afiliación sindical.

\subsection{Participación de los trabajadores}

En España, la participación de los trabajadores en los procesos electorales para designar a sus representantes en los órganos unitarios de empresa, puede considerarse bastante elevada (ver tabla 5); en otras palabras, allí donde los asalariados tienen la oportunidad de pronunciarse, suelen participar y dar su voto. Asimismo, se observa que, en el período de declive, posterior a 2007, el total de trabajadores y de empresas disminuye, así como la participación, mientras que aumenta el número de electores y de delegados. La representación es mayoritariamente masculina 59,9\% en 2015, pero aumenta notablemente la femenina que ha pasado del $21,4 \%$ de 2003 al 37,5\% en 2015. Entre los delegados predominan las edades centrales (35-49 años) puesto que representan el 45,8\%, seguidos de los delegados de 50 o más años $(29,6 \%)$, siendo los delegados más jóvenes (hasta 34 años) sólo el 11,1\%.

Dos de cada tres asalariados en empresas de 6 o más trabajadores no votan en las elecciones sindicales, en su gran mayoría por no tener posibilidad de hacerlo; ya que trabajan en empresas en las que no se han convocado elecciones. Esta es una fuerte debilidad, sobre todo si lo unimos al conjunto de empresas de menos de 6 trabajadores. De manera más ajustada, la proporción de trabajadores convocados a elecciones sindicales entre 2003 y 2015, y que se abstiene de participar, se cifra entre el $30 \%$ (hasta 2007) y el 35\% en 2015. La crisis del empleo, pues, y su precariedad perjudican los niveles de participación. 
Tabla 5. Asalariados según ámbito (en millones) y participación en las elecciones sindicales en España

\begin{tabular}{|c|c|c|c|c|}
\hline & 2003 & 2007 & 2012 & 2015 \\
\hline Trabajadores del ámbito privado $^{1}$ & 12,43 & 14,73 & 12,50 & 12,30 \\
\hline Trabajadores sin opción a $\mathrm{RS}^{2}$ & 1,88 & 2,16 & 1,96 & 2,01 \\
\hline Trabajadores en empresas 6 o más ${ }^{3}$ & 10,55 & 12,56 & 10,53 & 10,29 \\
\hline $\begin{array}{l}\text { Trabajadores en empresas } 6 \text { o más no convo- } \\
\text { cados }^{4}\end{array}$ & 4,53 & 5,90 & 3,76 & 3,73 \\
\hline Total electores ${ }^{5}$ & 6,02 & 6,66 & 6,77 & 6,56 \\
\hline Total votantes ${ }^{5}$ & 4,29 & 4,69 & 4,60 & 4,23 \\
\hline$\%$ electores s/ trabajadores ámbito privado & $48,4 \%$ & $45,2 \%$ & $54,2 \%$ & $53,3 \%$ \\
\hline$\%$ electores s/ trabajadores empresas 6 o más & $57,1 \%$ & $53,0 \%$ & $64,3 \%$ & $63,8 \%$ \\
\hline$\%$ votantes s/ trabajadores ámbito privado & $34,5 \%$ & $31,8 \%$ & $36,8 \%$ & $34,4 \%$ \\
\hline $\begin{array}{l}\% \text { votantes s/ trabajadores empresas } 6 \text { o más } \\
\text { trabajadores }\end{array}$ & $40,7 \%$ & $37,3 \%$ & $43,7 \%$ & $41,1 \%$ \\
\hline
\end{tabular}

Fuentes y notas: ${ }^{1}$ Asalariados afiliados a la SS; ${ }^{2}$ Trabajadores asalariados de empresas de hasta 5 trabajadores (sin representación sindical: RS); ${ }^{3}$ Asalariados no funcionarios en empresas de 6 o más trabajadores; ${ }^{4}$ Trabajadores en empresas de 6 o más no convocados a elecciones sindicales; ${ }^{5}$ Fuente SIC-CCOO según actas vigentes a fines de cada año.

Como resultado relevante cabe destacar que la participación se reduce conforme aumenta la plantilla de las empresas: en el año 2015, la participación en las empresas de menos de 50 trabajadores fue superior al 75\%, mientras que en las de más de 500 no alcanzó el 60\%. La mayor participación en las pequeñas empresas se debe quizás a la proximidad entre representantes y representados; aunque como hemos comentado pocas de ellas celebran elecciones sindicales, lo que constituye un importante reto.

La menor participación en las empresas de mayores dimensiones posiblemente responda a un mayor alejamiento de la acción sindical, a la complejidad derivada de situaciones de empleo muy variadas, o a la pluralidad de centros de trabajo. Profundizar en los motivos de baja participación en estas grandes empresas es, pues, un reto sindical. Con todo, la concurrencia de diversas opciones sindicales en una empresa posiblemente sea un estímulo para que los sindicatos hagan más visible su presencia en la misma, pero también puede revertir en una politización de la concurrencia sindical y la división puede incentivar la abstención.

\section{La presencia de diferentes opciones sindicales}

En 2015 UGT y CCOO suman el 69\% de los delegados. Muy lejos queda el resto de las agrupaciones sindicales (generales, corporativos, nacionalistas y otros sin- 
dicatos $)^{9}$ que organizan cada una de ellas entre el 6 y el $8 \%$ de los delegados. Las candidaturas no sindicales quedan en un testimonial $2,5 \%$.

En los años de crecimiento del empleo, hasta 2007, todas las opciones sindicales aumentan en número de delegados, con la única excepción de las candidaturas no sindicales. Pero con el cierre de empresas, la destrucción de empleo y la crisis posterior a 2007, el número total de representantes elegidos se reduce en un $16,9 \%$, afectando especialmente a los sindicatos mayoritarios, tanto de nivel estatal $(-23,2 \%$ en el caso de CC.OO y $-25,8 \%$ en el de UGT) como autonómico (-19,5\% para el conjunto de las candidaturas nacionalistas), mientras que las opciones minoritarias de tipo generalista ven reducida su representación en un $-3,6 \%$, registrándose un significativo incremento de las opciones corporativistas $(+8,9 \%)$ y no sindicales $(+3,8 \%)$, así como una mayor fragmentación $(+43,7 \%)$ para el conjunto de "otros" sindicatos.

Tabla 6. Distribución de los delegados sindicales según representación

\begin{tabular}{|ccccc|}
\hline & 2003 & 2007 & 2012 & 2015 \\
CCOO & 38,8 & 38,8 & 37,4 & 36,1 \\
UGT & 36,5 & 36,9 & 35,1 & 32,9 \\
Sind. generales & 5,0 & 5,0 & 5,6 & 5,8 \\
Sind. corporativos & 6,0 & 6,1 & 7,3 & 8,0 \\
Sind. nacionalistas & 6,3 & 6,5 & 6,3 & 6,3 \\
Otros sindicatos & 4,7 & 4,8 & 6,3 & 8,3 \\
No sindicales & 2,7 & 2,0 & 2,0 & 2,5 \\
Total delegados & 100,0 & 100,0 & 100,0 & 100,0 \\
\hline
\end{tabular}

Fuente: SIC-CCOO

La presencia de representantes de los trabajadores aumenta conforme lo hace el tamaño de la empresa. Sin embargo, si nos atenemos exclusivamente a las empresas con representación sindical, en estos casos en la pequeña empresa, y en términos comparativos, están presentes en mayor medida las opciones de clase mayoritarias, CCOO y UGT, también los sindicatos nacionalistas. Este argumento rompería con la idea de que estos grandes sindicatos sólo representan a los trabajadores más establecidos y seguros, es decir, los asalariados de las empresas de mayor dimensión. En 2015, el tándem CCOO y UGT representan el 73,5\% de los delegados en las empresas de menos de 500 trabajadores, llegando al 77-80\% en las de menor tamaño (de 6 a 9 y de 10 a 49 trabajadores). En las de mayor tamaño (de 500 o más trabajadores) estas dos opciones sólo alcanzan el 57,2\% de los delegados. Denotemos que las opciones sindicales corporativas y el resto de sindicatos alcanzan, conjuntamente, el $30 \%$ de la representación en las empresas de 500 o más trabajadores.

Naturalmente, conforme aumenta la plantilla de una empresa se reduce enormemente la posibilidad de que una opción sindical obtenga por sí sola mayoría en el órgano de representación unitaria de los trabajadores. Así, sólo en el 15,5\% de las empresas con representación sindical de 50 a 249 electores ninguna opción sindical

En nuestro estudio se ha ordenado a los distintos sindicatos en base a las siguientes categorías: minoritarios generalistas (USO, CGT, SU, SI), corporativos (CSIF, FSIE, ANPE, STEs, CEMSATSE, FETICO, FASGA, SEPLA, etc.) y nacionalistas (ELA-STV, LAB, CIG, IC, CSC). 
obtiene mayoría, mientras en las de 250 a 499 electores en un $31,8 \%$ no hay una opción sindical mayoritaria, o en el $50,1 \%$ de las de 500 o más electores. Esta ausencia de mayorías netas en las grandes empresas refleja la mayor competencia entre opciones sindicales y unos órganos unitarios de representación funcionando posiblemente con una dinámica próxima a la parlamentaria. Ello puede conducir a una visión más corporativa y centrada en la empresa, de la que es buena muestra el hecho de que otros sindicatos y los sindicatos corporativos obtengan sus mejores resultados en las empresas más grandes, y con gran diferencia con respecto a la conseguida en las de tamaños menores. De hecho, los que hemos agrupado como otros sindicatos siguen una distribución inversa a todas las demás opciones, dado que su porcentaje de representación es mayor conforme aumenta el tamaño de la empresa; mientras los sindicatos generales y los corporativos obtienen sus mejores resultados en las empresas de dimensiones intermedias.

\section{Discusión y conclusiones}

Las relaciones laborales en España se han configurado sobre la base de una representación de los trabajadores centrada en la audiencia (elección) y no en la presencia (afiliación). Dicha base electoral se fija institucionalmente en función de la dimensión de la empresa o centro de trabajo, una cuestión que determina el conjunto. No obstante, dicha determinación estructural sobre la representación (ejemplificada también para el caso de las empresas nuevas respecto de las que permanecen), queda matizada e incluso contrapesada por la acción sindical. De manera que allá donde hay afiliación hay más representación. Así, según la ECVT, la tasa de cobertura de la representación sindical es del $47,4 \%$, pero ésta varía entre el $75,4 \%$ allá donde hay afiliados a algún sindicato, y el $40,9 \%$ allá donde no hay afiliados. De este modo las diferencias de representación, participación, o de cobertura negociadora son sustanciales entre la población asalariada, entre asalariados con delegados o, sobre todo, con comité de empresa, respecto de los asalariados sin órganos colectivos de representación, así como entre asalariados con sindicatos en su empresa o sin la presencia de los mismos. En este sentido el número total de delegados, encargados finales en las empresas de las funciones de representación, pero también de negociación colectiva, es un claro ejemplo de la importancia del mecanismo electoral en las relaciones laborales españolas, comparativamente a otros sistemas (ver Hege et al., 2011, Chang, 2005 o Simms, 2011).

Por otra parte, los resultados son contundentes en torno al hecho de que conforme aumenta la dimensión de empresa hay más representación y afiliación, aunque no más participación en los procesos electorales. El factor estructural o institucional influye, hecho remarcado también por Farber (2001), Charlwood y Forth (2011) o Artus (2013). También incide la dinámica o demografía empresarial, pues las empresas de nueva creación tienen mayores dificultades para disponer de representación sindical que las empresas de más larga trayectoria.

Además, los sectores con más representación (servicios financieros, servicios públicos, transportes y comunicaciones e industria) son los que asimismo tienen más afiliación; en sentido contrario actúan los servicios personales. Idéntica secuencia siguen los contratos indefinidos (más representación y afiliación) respecto de los 
temporales. En términos de diferencias sectoriales o de tipos de contrato, los resultados se asemejan a los de Artus (2013). También a los de Charlwood y Forth (2011) que destacan que la vinculación entre presencia afiliativa y representación es más acusada en la industria y en el sector público que en los servicios privados. Y dentro de estos últimos Artus (2013) destaca la distancia entre la representación en la industria de la energía o en los servicios financieros y las modestas cifras en hostelería, restauración y servicios personales.

Observados estos datos desde el punto de vista opuesto, es decir desde el análisis de conjunto de las empresas sin representación, ello permite establecer que la probabilidad de que una empresa carezca de representación es mayor cuando estamos ante una empresa de nueva creación con muy poca plantilla, de actividades de servicios y sin afiliación sindical; lo que encajaría con la vertiente negativa de la hipótesis de Farber (2001), en el sentido de un cambio de actitud empresarial hacia los sindicatos. Cambio que obvia los efectos positivos que las relaciones laborales cooperadoras pueden suponer en términos de productividad (Bryson et al., 2006), de innovación técnica (Bryson et al., 2005) o de resolución de problemas laborales (Antcliff y Saundry, 2009).

Pero hay factores contextuales que también influyen. Así, la representación sindical (medida en número de delegados) ha seguido el ritmo de los ciclos económicos. Esto nos lleva a dos consideraciones. La primera es la constatación de que a partir de 2007 ha descendido el número de delegados; y la segunda nos conduce a un interrogante, sobre si ese descenso responde sólo a los efectos de la caída del empleo y cierre de empresas, o bien incorpora también rasgos del declive continuado que Farber (2001) dibuja para el caso norteamericano o Charlwood y Forth (2011) para el Reino Unido.

La literatura especializada muestra que, aunque hay factores transversales como los ya reseñados -tamaño de empresa o centro de trabajo y sector principalmente-, la representación sindical en los diferentes países europeos se configura en función de los grandes modelos de estado del bienestar y de relaciones laborales; hasta el punto de que incluso la valoración u opinión sobre dicha representación se vincula a la división entre sistemas nórdico, centroeuropeo, anglosajón, latino y países del Este (Beneyto, 1998; Hyman, 2001; EIRO, 2007). Por ello, no sólo es importante el factor contextual (tipo de empresa y sector), sino también la incidencia del marco institucional en la estructura y dinámica final del sistema de representación sindical adoptado en un país. Dichos elementos institucionales dependen de las leyes, acuerdos y convenios adoptados y, en España, concretamente la representación de los asalariados en las empresas se construyó sobre la base del ET y de la LOLS, con las modificaciones posteriores correspondientes. Aunque no deben descuidarse las oportunidades que abre a la representación de los asalariados la acción colectiva impulsada desde las organizaciones sindicales.

Si el punto de partida son las unidades empresariales, en España quedan excluidas de la representación (por tener menos de 6 trabajadores) cerca de un millón de empresas en 2015 (una buena parte de ellas son de uno o dos empleados) -aunque hasta el momento los convenios colectivos de ámbito supraempresarial les sirven como referencia contractual; es decir, que los sindicatos, en función de los delegados elegidos en las empresas también les representan, aunque indirectamente. Incluso entre las 286.814 empresas que pueden realizar elecciones, la mayoría (90\%) son de dimensión reducida (de 6 a 49 trabajadores). Apenas el 2\% de las empresas tiene la 
posibilidad de tener comité de empresa por superar los 50 asalariados y es en ellas donde están presentes la mayor parte de los 6.557 .859 electores. Ahora bien, puesto que la participación en las elecciones sindicales es del 64,5\%, puede decirse que se trata de un sistema de representación de los trabajadores asalariados que funciona con relativa eficiencia, representatividad y legitimidad.

Sin embargo, uno de los aspectos de las relaciones laborales españolas, que recordemos nacieron fruto de un acuerdo en los inicios de la transición a la democracia, es que institucionaliza un sistema de representación basado en la dimensión de la empresa. Esto ha permitido una representación amplia, puesto que los convenios colectivos han cubierto un buen porcentaje de asalariados, aunque las recientes reformas pueden dar al traste con ello. El mecanismo, además, ha funcionado desde el punto de vista de los asalariados, puesto que casi un 50\% de ellos está, de un modo u otro, implicado, y de éstos casi un $70 \%$ participa activamente. Pero como cualquier sistema, no está exento de crítica o perfeccionamiento, aunque desde la contraparte empresarial, y también desde gobiernos de distinto signo, a veces se ha obviado la base de la legitimación del sistema de representación sindical español (las elecciones) para fijarse en las más modestas cifras de la afiliación, no precisamente incentivada por el modelo de audiencia diseñado en los inicios de la transición, ni tampoco por las cláusulas "erga omnes" de la negociación colectiva, que implican el beneficio automático de las mejoras del convenio a todos los trabajadores con independencia de su adscripción o no a un sindicato.

Desde el punto de vista intrasindical, se debe señalar que en cuanto a la representación de los asalariados se detectan diversas tendencias generales. Por una parte, hasta finales de la década de los 80 se observa una alternancia entre UGT y CCOO en la posición más votada; recordemos, son años de fuerte competencia entre ambas centrales sindicales. En ese periodo y hasta el inicio de la crisis económica de 2007, ambos sindicatos agrupan entre el $75-80 \%$ de la representación sindical; una representatividad que en el año 2015 reducen al 69\%. Las otras opciones sindicales (corporativos, generales, nacionalistas y otros sindicatos), sobre todo los primeros y los últimos, logran crecer en diversos ámbitos, sin descartar la variación en la composición de empresas y del empleo en dicho periodo.

La propensión a la participación o la representación entre los asalariados se relaciona con las oportunidades que ofrecen las organizaciones y, entre ellas, no sólo está la organización, sino también la información y la comunicación, un apartado en el que hay mucho trabajo por hacer. Así, la satisfacción de los asalariados con el sistema de representación es sumamente contradictoria (ECVT, 2010). Por una parte, hay un fuerte desconocimiento sobre la existencia de representación sindical y más aún de convenio colectivo; por otra, se valora positivamente el convenio -fruto de la acción de los sindicatos-, mientras se valora menos a las organizaciones representativas que lo hacen posible. Dicha valoración cambia de manera notoria en función de la proximidad de los asalariados al sindicato (información, afiliación), aunque en general aumenta con el tamaño de la empresa, el nivel educativo, la antigüedad en la empresa y la estabilidad del contrato. El conocimiento de la actividad sindical y la proximidad abren vías de mejora del nivel de satisfacción y de un mayor compromiso sindical. De hecho, según los datos SIC-CCOO manejados, en las empresas de menores dimensiones, así como en aquellas de dimensiones medias o grandes (excepto las de más de 500 electores), y en las unidades productivas con mayor afiliación sindical, la participación tiende a ser más elevada; mientras la precariedad 
en el empleo, que afecta sobre todo a jóvenes y a mujeres, y la mayor presencia de afiliación reciente o de asalariados de actividades de servicios de bajo salario, perjudica los niveles de participación. En este sentido, ver Artus (2013) para el caso alemán. Otro elemento clave es el reseñado por Charlwood y Forth (2011) al remarcar la distancia (en términos demográficos -sexo, edad, instrucción- y ocupacionales -contrato, antigüedad en la empresa) entre el perfil tipo del delegado con experiencia y la presencia diferenciada entre los asalariados de un mayor número de jóvenes, mujeres, temporales, desempleados, etc.

En definitiva, el sistema de representación sindical en España por medio de las elecciones sindicales es democrático, abierto, flexible y su consolidación en un período convulso en términos económicos (dos fuertes crisis y una menor) y políticos (fin de la dictadura), pero también ideológicos, dado que el neoliberalismo justamente pretende acabar con las regulaciones en el ámbito laboral y, naturalmente, minimizar sindicatos y negociación colectiva, es muestra de su capacidad de resistencia e incidencia. Volviendo a la hipótesis inicial podemos decir que el sistema de representación dual en España adquiere rasgos de complementariedad (entre el canal sindical y el unitario), pero también de competencia, no sólo entre los mismos sindicatos, sino también entre las organizaciones sindicales y la representación unitaria, lo que favorece el efecto free-rider.

Por ello es un sistema perfectible. En primer lugar, requiere de unas reglas del juego claras. Si se trata de mantener la democracia y sociedades inclusivas y no excesivamente desiguales, basadas en una redistribución justa, son necesarias voces plurales (los sindicatos) ante la exclusividad y la discrecionalidad de una sola voz (la empresarial), pero también mecanismos equilibradores y reguladores como las leyes protectoras, la capacidad negociadora, las normas pactadas en convenios colectivos, la misma Seguridad Social. Las elecciones sindicales en España han permitido la existencia de sindicatos y la expansión de la negociación colectiva. En términos afiliativos sería deseable la adecuación entre afiliación y elección, porque en el fondo los desequilibrios debilitan la representación de los asalariados y ello requiere cambiar la estructura de oportunidades y de acción de los sindicatos españoles; también ciertamente la falta de articulación y de coordinación negociadora es un obstáculo comparando los resultados laborales con otros países de negociación más coordinada. En todo caso, en el momento actual del sistema de relaciones laborales y de la democracia en España, es necesario frenar la ofensiva neoliberal y proponer un nuevo contrato social. Y ello no es una cuestión exclusivamente social, sino también política, y de carácter acuciante.

\section{Bibliografía}

Antcliff, V.; Saundry, R. (2009). “Accompaniment, Workplace Representation and Disciplinary Outcomes in British Workplaces. Just a Formality?". British Journal of Industrial Relations, 47 (1): 100-121.

Artus, I. (2013). "Precarious delegates: irregular forms of employee interest representation in Germany". Industrial Relations Journal, 44 (4): 409-424.

Baylos, A. (1991). "La intervención normativa del Estado en materia de relaciones colectivas". En F. Miguélez y C. Prieto (coords.), Las relaciones laborales en España (pp 
289-306). Madrid: Siglo XXI.

Baylos, A. (2012). "El sentido general de la reforma: la ruptura de los equilibrios organizativos y colectivos y la exaltación del poder privado del empresario en la Ley $3 / 2012$ ”. En A. Baylos (dir.), Políticas de austeridad y crisis en las relaciones laborales: la reforma del 2012. Albacete: Bomarzo.

Beneyto, P.J. (2008). "El sindicalismo español en perspectiva europea: de la anomalía a la convergencia". Cuadernos de Relaciones Laborales, 26 (1): 57-88.

Blaschke, S. (2011). "Determinants of female representation in the decision-making structures of trade unions". Economic and Industrial Democracy, 32 (3): 421-438.

Bryson, A.; Forth, J.; Kirby, S. (2005). "High-Involvement Management Practices, Trade Union Representation and Workplace Performance in Britain". Scottish Journal of Political Economy, 52 (3): 451-491.

Bryson, A.; Charlwood, A.; Forth, J. (2006). "Worker voice, managerial response and labour productivity: an empirical investigation". Industrial Relations Journal, 37 (5): 438-455.

Chang, T.F.H. (2005). "Local Union Leaders' Conception and Ideology of Stewards' Roles". Labor Studies Journal, 30 (3): 49-71.

Charlwood, A. ; Forth, J. (2011). "Les mutations de la représentation des salariés en Grande-Bretagne". Revue de l'IRES, 68 (1): 43-73.

EIRO (2007). Industrial relations in the EU, Japan, US and the other global economies, 2005-2006. Dublin.

Farber, H.S. (2001). “Union Success in Representation Elections: Why Does Unit Size Matter?". Industrial and Labor Relations Review, 54 (2): 329-348.

Freeman, R. (2007). "Do Workers Still Want Unions? More than Ever”. EPI Briefing Paper, 182. Washington, DC: Economic Policy Institute, American Workplace.

Gumbrell-McCormick, R.; Hyman, R. (2006). "Embedded collectivism? Workplace representation in France and Germany". Industrial Relations Journal, 37 (5): 473-491.

Hege, A.; Lévesque, C. ; Murray, G. ; Dufour, C. (2011). "Les délégués, acteurs stratégiques du renouveau syndical?». Revue de l'IRES, 68: 4-18.

Hyman, R. (2001). Understanding European Trade Unionism. Between Market, Class \& Society. London: SAGE Publications.

Köhler, H.D.; Calleja, J.P. (2011). "Retos de futuro para las organizaciones sindicales en España”. Gaceta Sindical. Reflexión y debate, 16: 119-138.

Le Capitaine,C.; Lévesque, C.; Murray, G. (2011). “ Contre vents et marées : le délégué au cœur du renouveau syndical». Revue de l'IRES, 68 (1): 142-171.

Lévesque, C.; Murray, G. (2010). "Comprendre le pouvoir syndical: ressources et aptitudes stratégiques pour renouveler l'action syndicale”. Revue de l'IRES, 65 (2): 41-65.

Olson, M. (1992). La lógica de la acción colectiva. México: Limusa.

Rigby, M.; Contrepois, S.; O’Brien Smith, F. (2009). “The Establishment of Enterprise Works Councils: Process and Problems". European Journal of Industrial Relations, 15 (1): 71-90.

Simms, M. (2011). "Les militants de terrain dans les campagnes d'implantation syndicale dans le secteur des services en Grande-Bretagne». Revue de l'IRES, 68 (1): 75-108.

Taras, D.G.; Kaufman, B.E. (2006). "Non-union employee representation in North America: diversity, controversy and uncertain future". Industrial Relations Journal, 37 (5): 513-542.

Valdés Dal-Ré (2013): “La reforma laboral de 2012”. Relaciones laborales: Revista crítica de teoría y práctica, 2: 1-10. 